\title{
Impacto y recomendaciones de clase invertida en el proceso de enseñanza-aprendizaje de geometría
}

\author{
Jocelyn A. Albornoz-Acosta(1), José G. Maldonado-Cid(2), Cristian L. Vidal-Silva ${ }^{(3) \star}$ y Erika Madariaga ${ }^{(4)}$ \\ (1) Ingeniería en Metalurgia, área de Minería y metalurgia, Sede Calama, Universidad Tecnológica de Chile INACAP, \\ Granaderos 3250, Calama - Chile. (e-mail: jocelyn.albornoz@gmail.com) \\ (2) Ingeniería en Metalurgia, área de Minería y metalurgia, Sede Calama, Universidad Tecnológica de Chile INACAP, \\ Granaderos 3250, Calama - Chile. (e-mail: jose.maldonado31@inacapmail.cl) \\ (3) Departamento de Administración, Facultad de Economía y Administración, Universidad Católica del Norte, \\ Antofagasta - Chile. (e-mail: cristian.vidal@ucn.cl) \\ (4) Facultad de Ingeniería, Ciencia y Tecnología, Universidad Bernardo O’Higgins, Avenida Viel 1497, Ruta 5 Sur, \\ Santiago - Chile. (e-mail: erika.madariaga.garcia@gmail.com)
}

* Autor a quien debe ser dirigida la correspondencia.

Recibido Jun. 19, 2019; Aceptado Ago. 20, 2019; Versión final Sep. 30, 2019, Publicado Jun. 2020

\begin{abstract}
Resumen
Este artículo presenta la planificación y aplicación del modelo pedagógico de clase invertida para la enseñanza-aprendizaje de geometría. La metodología de clase invertida es una alternativa para el desarrollo de competencias en estudiantes trabajadores y no trabajadores de pregrado, y representa una alternativa adecuada para el desarrollo de competencias y aprendizajes esenciales como la geometría. En Chile, la geometría es parte de la línea curricular matemática base en diferentes áreas de formación profesional para el desarrollo de competencias esenciales. Este estudio se realizó con 176 estudiantes chilenos de primer año de las carreras de ingeniería en mina e ingeniería en metalurgia que cursaban la asignatura de geometría. Los resultados muestran mejoras en rendimiento académico de estudiantes no trabajadores y en estudiantes trabajadores, y comparando ambas mejoras, se obtiene un desempeño académico superior de los primeros. Así, este trabajo demuestra la aplicabilidad de la clase invertida para la educación de estudiantes trabajadores.
\end{abstract}

Palabras clave: clase invertida; aprendizaje; geometría; impacto; recomendaciones

\section{Flipped classroom impact and recommendations in the teaching-learning process of geometry}

\begin{abstract}
This article presents the planning and application of the inverted class pedagogical model for teaching-learning of geometry. The inverted class methodology is an alternative method for skill development in working undergraduate students and in non-working undergraduate students, and represents an adequate option for developing competences and essential learning such as geometry. In Chile, geometry is part of the basic mathematical curricular line in different areas of professional education for the development of essential skills. This study was conducted on 176 first year mining engineering students and metalurgic engineering students that were taking a geometry class. The results showed improvements in the academic performance of both non-working students and working-students, and comparing both improvements, higher academic performance is obtained from the former. Thus, this work demonstrates the applicability of the inverted class for the education of working students.
\end{abstract}




\section{INTRODUCCIÓN}

Tal y como lo señala Crompton et al. (2018), la geometría se corresponde con el estudio de las propiedades, relaciones y transformaciones de objetos espaciales en un sistema definido. Según Clement (2004), la geometría y el razonamiento espacial son una base para el aprendizaje de las matemáticas y el desarrollo de otras ciencias tales como diversas especialidades de la ingeniería. De esta forma, tanto la geometría como el razonamiento espacial son de gran relevancia en el proceso formativo de las personas. Además, hay ejemplos relativamente actuales que reafirman la importancia de la geometría y el espacio para la formación de futuros profesionales (Boccaccio et al., 2016; Prasad y Vijayendra, 2017). En Chile, respecto a la relevancia de la geometría, diversas carreras de educación superior consideran la geometría como elemento base en sus tópicos y áreas de formación (Friz et al., 2018). Por ejemplo, INACAP (2019) considera el estudio de geometría como parte base de asignaturas en los programas de estudio de carreras profesionales y técnicas de las áreas de construcción, informática, minería y metalurgia, en la búsqueda del desarrollo de competencias para con la aplicación de fundamentos de geometría descriptiva, geometría analítica y trigonometría plana en la resolución de problemas propios de cada especialidad.

Respecto al estudiante-trabajador, por ejemplo, la institución INACAP (2019) cuenta con un 41\% de estudiantes trabajadores, es decir, estudiantes que sólo asisten como mínimo a un $50 \%$ de clases presenciales lo que provoca una discontinuidad en el proceso de enseñanza-aprendizaje de estos estudiantes. Los estudiantes-trabajadores son una tendencia actual en el mundo principalmente por la masificación de la educación superior, entonces es muy frecuentes encontrar estudiantes universitarios que trabajan tanto en modalidad tradicional de estudios (jornada diurna) o en jornada vespertina (Sjoberg-Tapia et al., 2018). Además, en gran medida, muchos de estos estudiantes-trabajadores requieren actualizar sus bases tecnológicas con el fin de aprovechar las ventajas de las mismas en su proceso formativo (CamachoYáñez et al., 2015).

Respecto a metodologías de enseñanza-aprendizaje para el desarrollo de competencias para con los estudiantes, el modelo pedagógico de clase invertida requiere un rol diferente del estudiante, esto es, ya no como mero receptor de las clases (Bergmann y Sams, 2014). Las principales características de una clase invertida son: 1) un entorno flexible (acceso claro y natural de los participantes); 2) cultura del aprendizaje (valoración de contenidos por medio de un escalonado cambio de roles); 3) contenido dirigido (videos editados por el profesor con la inclusión de contenidos más prioritarios); y 4) el profesor como guía (el profesor guía a los estudiantes en el aula para solucionar dudas y así los estudiantes aprenden). Claramente, se requiere de un compromiso de participación del estudiante en cada clase, para que el proceso de enseñanza- aprendizaje sea efectivo; actitud usualmente propia de personas con altos niveles de responsabilidad como lo son los estudiantes-trabajadores. De esta forma, el modelo pedagógico de clase invertida representa un gran cambio de roles para los profesores y los alumnos, ya que presenta diversas ventajas potenciales en su aplicabilidad respecto al aprendizaje de los estudiantes (Novak et al., 2016; Arráez et al., 2018).

Por los antecedentes ya expuestos, esto es, por la relevancia de la geometría en la formación profesional, por el alto grado de estudiantes-trabajadores existentes en la actualidad en Chile, y por los beneficios potenciales del modelo pedagógico de clase invertida en el proceso enseñanza-aprendizaje, el principal objetivo de este trabajo es presentar el impacto y recomendaciones de los resultados de la aplicación de clase invertida como metodología de aprendizaje activa y colaborativa en el aula en el proceso de enseñanza-aprendizaje en la asignatura de geometría, para así lograr un aprendizaje significativo de los alumnos principalmente estudiantes-trabajadores.

\section{GEOMETRÍA}

La geometría (medir la tierra) es una rama de las matemáticas que, según Orozco (2014), tiene sus orígenes en el antiguo Egipto y en Grecia con Pitágoras y Euclides (geometría Euclidiana). Es una parte de la matemática para el estudio de las propiedades y medidas de figuras en un plano o espacio potencialmente útiles para la representación o modelamiento de fenómenos en otros contextos.

\section{Geometría y Educación}

La riqueza de la geometría en educación es su estrecha relación con otros dominios matemáticos tales como las ciencias naturales y sociales. En la dimensión biológica, la geometría se relaciona con capacidades humanas como el sentido espacial, con la percepción y visualización. En la dimensión física, la geometría indaga por propiedades espaciales y representación de los objetos físicos y modelar el espacio circundante. En su dimensión teórica, la geometría integra la abstracción y rigor de diversas teorías ya que esta constituye una herramienta de representación e interpretación de otras ramas del conocimiento. Por la experiencia de educandos y educadores, los modelos en forma de figuras, tablas o diagramas juegan un rol muy relevante en los procesos de aprendizaje y desarrollo de la ciencia. Tal y como lo resaltan Rüde et al. (2018) y (Dimmed 
y Herbert, 2018), las abstracciones geométricas proveen ideas, estructuras y categorías además de funciones, relaciones, algoritmos y axiomas que constituyen la base de cualquier teoría, esto es, modelos que muy utilizados como pruebas prácticas de consistencia. Según Velishova (2002), la geometría es de alta relevancia para el razonamiento abstracto necesario para la construcción de modelos de la realidad, más aún hoy en día, por la alta presencia de computadores y modelos computacionales con la base de diferentes figuras geométricas, además de herramientas computacionales y trabajos de investigación del área de sistemas informáticos como apoyo del proceso enseñanza - aprendizaje de la geometría (Calderon, 2015; Diaz-Nunja et al., 2018; Ferreira de Souza, 2017; Fonseca-Pérez y Gamboa-Grau, 2010).

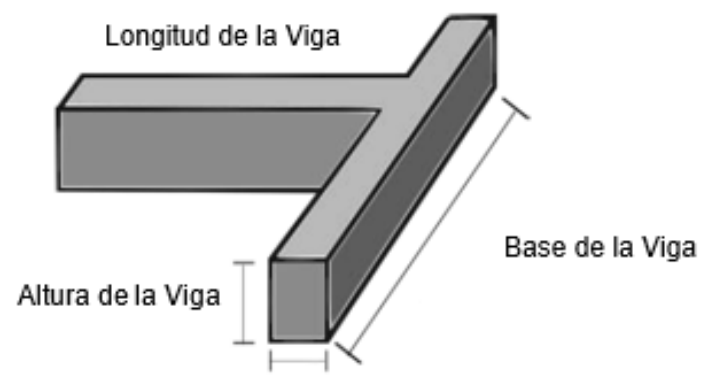

Fig. 1: Figura geométrica ejemplo de uso en ingeniería.

Según Dimma y Herbert (2018), la geometría representa un primer acercamiento para estudiantes de educación secundaria a las demostraciones matemáticas con figuras y modelos geométricos; una evidencia más de la aplicabilidad teórico- práctica de la geometría para futuros ingenieros en la resolución de problemas. Por ejemplo, la figura 1 modela una situación propia de ingeniería para determinar la cantidad de material a usar según las dimensiones, el área y/o volumen, del mismo.

Respecto a las dificultades para el aprendizaje de la geometría, Alikia et al. (2017) menciona ideas referentes a la falta de capacidad de abstracción en los jóvenes actuales para poder visualizar el uso de figuras geométricas básicas como medio de representación de la realidad; por ejemplo, el uso y alcance de figuras rectangulares.

\section{Aplicaciones de Geometría}

La geometría está presente en diversas áreas de la vida humana, no necesariamente académica tales como arte, arquitectura y física: 1. Según Gamwell (2015), la geometría y el arte están muy relacionados principalmente en la teoría de la perspectiva que representa el origen del concepto de proyectivo; 2 . Según Pottmann et al. (2008), la geometría es la base de todo proyecto de arquitectura; 3. Existe una visión histórica previo a Newton, en la época de Newton y posterior a Newton (Szekeres, 2004) de la relación entre la geometría y la física.

\section{CLASE INVERTIDA}

El aula invertida (del inglés flipped classroom) se corresponde con un modelo pedagógico o un método de enseñanza-aprendizaje cuyo principal objetivo es que el alumno asuma un rol mucho más activo en su proceso de aprendizaje que el usado tradicionalmente, esto es, ya no es un simple receptor de contenidos (Bergmann y Sams, 2014). A grandes rasgos consiste en que el alumno estudie los conceptos a revisar en la siguiente clase antes de la misma por medio de diversas herramientas que el docente facilita, principalmente vídeos o audios digitales del contenido a revisar, y el tiempo de clase se aproveche para resolver dudas relacionadas con el material proporcionado, para la realización de prácticas y abrir foros de discusión sobre cuestiones controvertidas (Berenguer, 2016).

Las bases fundamentales del modelo de clase invertida se reflejan en cuatro premisas fundamentales las que sirven de anclaje para capitalizar la teoría en la práctica (Bergmann y Sams, 2014). Dichas premisas se corresponden con términos que comienzan con las letras de la palabra en inglés invertir (flip): F (Flexible environments, en español ambiente flexible), L (Learning culture, en español cultura de aprendizaje), I (Intentional content, en español contenido intencional), P (Professional educators, en español educadores profesionales) (Hamdam et al., 2013; Bergmann y Sams, 2014). Tal y como lo señalan Bergmann y Sams (2017), la metodología de clase invertida se corresponde con una estrategia educativa para innovar al interior del aula para un afianzamiento del conocimiento, teniendo en cuenta los procesos educativos que llevan los estudiantes. Tal como lo ilustra la figura 2 (Monitor, 2019), el modelo pedagógico de clase invertida busca 
proporcionar oportunidades para un aprendizaje integral mediante la utilización de la tecnología gracias a actividades dentro del aula con una alta participación de los estudiantes.

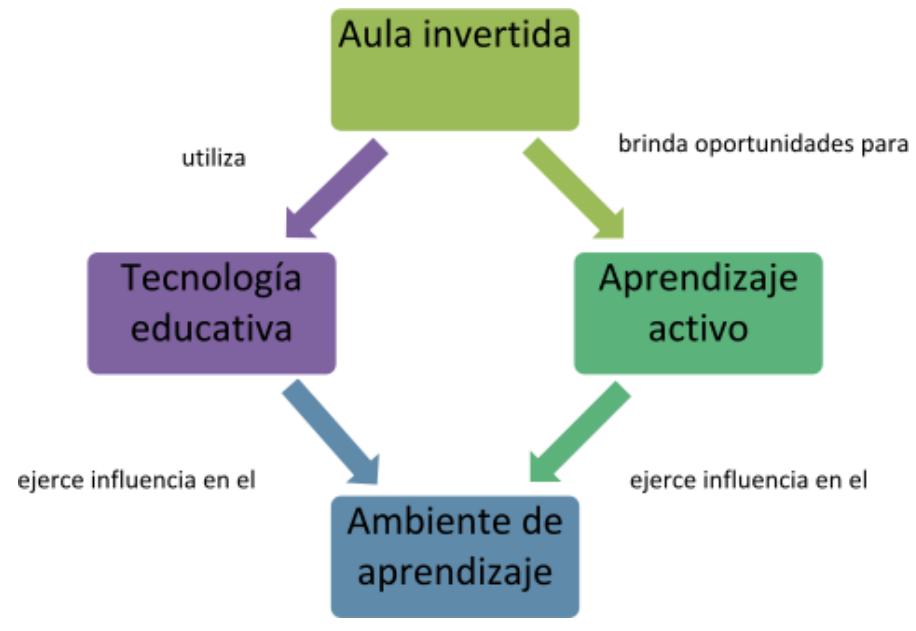

Fig. 2: Impacto e influencia de modelo pedagógico de clase invertida.

En la aplicación del modelo pedagógico de clase invertida, es necesario que el profesor cambie su rol de actor, por el de orientador, esto es, cambiar el rol de ser un mero expositor de conocimientos por el rol de asesor para así transferir el protagonismo a los alumnos (Perdomo, 2016; Tourón et al., 2014). En este contexto, una clase invertida permite al profesor moverse en un escenario de atención a determinados conocimientos, a la interpretación de los conocimientos para atender dudas y/o detalles de comprensión de los estudiantes para llevar a todos los participantes en el aula a su aplicación (Perdomo, 2016).

Tal y como lo argumenta (Tourón y Santiago, 2015), la metodología pedagógica de clase invertida es una herramienta óptima para el desarrollo de competencias de los alumnos más capaces. Además, la clase invertida permite ensenar al alumnado a sus ritmos individuales, lo que supone una personalización superior para cada uno de los participantes. Así, este modelo puede resultar idóneo para desarrollar talento en los estudiantes más capaces. Otra razón para potenciar la aplicación de esta metodología de enseñanza es que ella permite aumentar y hacer más efectivo el tiempo para el aprendizaje durante las clases. Justamente, por este último motivo, la clase invertida ha ganado popularidad en todas las áreas de formación y contenido. Por lo tanto, una buena clase invertida debería ser como cualquier otra en la que se lleve a cabo una buena enseñanza y un aprendizaje efectivo (Tourón et al., 2014).

\section{METODOLOGÍA}

La experiencia en estudio se realizó en el año 2016 con 176 estudiantes de primer año de las carreras de Ingeniería en Mina e Ingeniería en Metalurgia quienes cursaban la asignatura de Geometría. Para estos experimentos, se diseñaron guías de aprendizajes y se grabaron videos en formato mp4 con los contenidos de las distintas clases y ejercicios resueltos con explicaciones detalladas para que los estudiantes pudieran desarrollar los aprendizajes esperados fuera del aula. La implementación del proyecto se realizó en dos etapas: la primera se focaliza en determinar las necesidades de los estudiantes y el diseño de recursos de aprendizaje de acuerdo a esas necesidades detectadas, mientras la segunda se corresponde con la aplicación de los recursos diseñados y su implementación a través de la metodología de clase invertida para lograr un positivo impacto e influencia en el proceso de enseñanza-aprendizaje de los estudiantes participantes en el experimento.

\section{Fase de diseño de Recurso de aprendizaje}

En esta fase se llevaron a cabo las siguientes actividades: (1) Etapa de levantamiento de datos: se identificó, por medio de datos históricos de la asignatura, las mayores dificultades y falencias de los alumnos con respecto a la asignatura de Geometría, además de evaluar los desempeños históricos de alumnos en semestres anteriores para con dicha asignatura. Con el análisis de dichos antecedentes se escogió la cantidad de videos por unidad, así como el contenido de estos; y (2) Etapa de desarrollo del material didáctico de apoyo: se trabajó en el diseño de 9 guías de aprendizaje y en la grabación de 34 videos, con el propósito de lograr un aumento significativo en los indicadores de aprobación y promedio de notas. Los videos comprendían la implementación de actividades para la resolución de problemas, basado en el desarrollo del razonamiento lógico-matemático para así facilitar la interacción de los estudiantes con los ejercicios de contenidos en revisión. Las guías se diseñan con el propósito de trabajar tanto fuera como dentro 
del aula con la pretensión de que los estudiantes, luego de una explicación breve de los contenidos de los aprendizajes, con la observación de ejemplos resueltos con ejercicios desarrollados paso a paso, sean capaces de resolver de manera autónoma los ejercicios propuestos con una retroalimentación de sus resultados mediante la solución del problema.

\section{Fase de implementación de los recursos de aprendizaje}

Las actividades realizadas en esta fase fueron las siguientes: (1) Una entrevista inicial para conocer la percepción de los alumnos respecto a la asignatura de Geometría; en la cual se realiza a 176 estudiantes, de 8 sesiones que cursan la asignatura, en las jornadas: diurna y vespertina, utilizando una escala Likert: Totalmente en desacuerdo, en desacuerdo, indiferente, de acuerdo, totalmente de acuerdo (2). Se presentó el proyecto en cada una de las secciones de contenidos del curso, motivando a los alumnos a ingresar a la plataforma donde se encontraba el material disponible para descargar videos y guías de aprendizaje de cada unidad; (3) Los docentes que dictaron la asignatura de Geometría motivaron clase a clase a los alumnos a desarrollar las actividades y a revisar los videos; (4) En la metodología de trabajo, a los estudiantes se les asignó la tarea de ver y analizar los videos correspondientes a la siguiente clase, antes de la misma, de manera que los estudiantes llegaran a la siguiente clase con los contenidos estudiados. Con este estudio previo, cada profesor daba inicio a su clase conversando con los estudiantes para lograr la construcción del conocimiento a través de la formalización de los aprendizajes previamente revisados y potencialmente ya adquiridos por los estudiantes fuera del aula. En este mismo contexto, los profesores también aclaraban dudas y respondían consultas que emergían de la revisión previa de los videos. Posterior a esto y durante el desarrollo de la clase, a través de un trabajo colaborativo, los estudiantes resolvían las guías de aprendizajes. Para el cierre de la clase, se revisaban los ejercicios, reforzaban aprendizajes adquiridos y se indicaban de los videos que debían revisar para la clase siguiente. En el caso que los estudiantes no asistieran a clases presenciales, ellos desarrollaban las actividades desde su casa o lugar de trabajo para luego cargarlas al sistema en línea para la revisión y retroalimentación del profesor para estos estudiantes; (5) Se hicieron entrevistas presenciales en cada una de las secciones, para poder realizar una retroalimentación de parte de los alumnos a fin de buscar mejoras para con la realización del proyecto; y (6) Se realizó una entrevista final escrita, donde 100 alumnos de las 8 sesiones respondieron preguntas respecto a la experiencia de ser partícipes de este proyecto, indicando su opinión en la participación del proyecto a través de la escala Likert, donde debían responder en una escala de cinco puntos: Muy satisfecho, relativamente satisfecho, indiferente, relativamente insatisfecho y muy insatisfecho.

Mediante la aplicación de la metodología de clase invertida, como parte de las actividades ya descritas, el estudiante-trabajador estaba habilitado para adquirir los conocimientos y habilidades desarrolladas en las distintitas unidades del curso. Además, para los estudiantes que participaron y asistieron habitualmente a cases, existieron oportunidades de retroalimentación de los contenidos y aprendizajes esperados en cada sesión de clases. De esta forma, se optimiza significativamente el proceso de enseñanza-aprendizaje, ofreciendo al estudiante herramientas que le permiten desenvolverse con autonomía y así adquirir conciencia de su proceso de aprendizaje. Además, esta metodología de enseñanza-aprendizaje permite optimizar y apoyar académicamente al estudiante incentivando en ellos el desarrollo de competencias genéricas tales como la autogestión, el trabajo en equipo y la resolución de problemas.

\section{RESULTADOS}

A través del modelo de clase invertida junto con los recursos de aprendizajes diseñados y utilizados durante el año 2016, existió un incremento notable en el rendimiento de los estudiantes para cursos con los mismos tópicos pero con diferente metodología de enseñanza-aprendizaje tal y como lo refleja la tabla 1 (el año 2015 la metodología de enseñanza usada es la metodología tradicional donde el profesor es quien enseña y dirige la clase, mientras los estudiantes son principalmente receptores). Durante los años 2015 y 2016 , los alumnos rindieron 3 evaluaciones sumativas de desarrollo con énfasis en la resolución de problemas, además de 3 evaluaciones formativas y un examen final para aquellos estudiantes que no logran el promedio 5,0 con las evaluaciones del semestre.

Como se aprecia en la tabla 1, hay diferencias considerables tanto en la tasa de reprobación la que disminuye entre los años como en el promedio de notas el que aumenta. Todo esto, a pesar de la disminución en la tasa de asistencia. Dichos impactos serían principalmente por el posible acceso de los estudiantes a las clases desde cualquier lugar con acceso a Internet para utilizar recursos de aprendizaje como videos y también para el desarrollo de guías de aprendizaje.

En la figura 3 se observa la tasa de aprobación de la asignatura según diferentes periodos o semestres en los que se dictó, en la cual se verifica un aumento en el porcentaje desde semestre otoño 2015 a otoño 2016 en un 19\%, pero luego en el semestre primavera 2016 disminuye un 7,6\% con respecto a otoño 2016 . Esta 
disminución en la aprobación de la asignatura se debe principalmente a la deserción estudiantil durante dicho semestre. Con respecto a la satisfacción de los estudiantes y la evaluación del proyecto, de acuerdo a las distintas entrevistas realizadas, la tabla 2 resume la información obtenida.

Tabla 1: Comparación de los resultados

\begin{tabular}{|c|c|c|}
\hline Criterio & Primavera 2015 & Primavera 2016 \\
\hline $\mathrm{N}^{\circ}$ Estudiantes & 161 & 4,4 \\
\hline Promedio notas & 4,1 & $75,4 \%$ \\
\hline Porcentaje de aprobación & $57 \%$ & $75,5 \%$ \\
\hline Porcentaje de asistencia & $80,2 \%$ & \\
\hline
\end{tabular}

Porcentaje Aprobación en Curso de Geometría

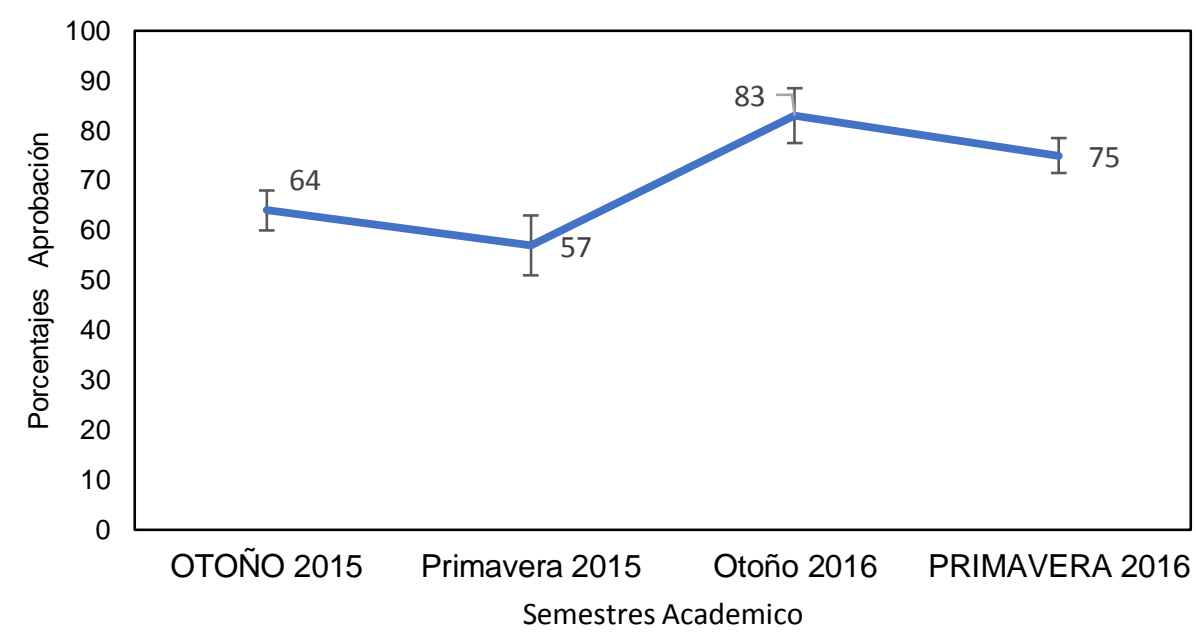

Fig. 3: Tasa de aprobación asignatura de geometría entre el 2015 y el 2016

Tabla 2: Resumen sobre la satisfacción de los alumnos con respecto al proyecto en otoño 2016

\begin{tabular}{|l|c|c|c|c|c|}
\hline $\begin{array}{l}\text { Al finalizar la asignatura, } \\
\text { ¿cuáles son los } \\
\text { sentimientos? }\end{array}$ & Muy satisfecho & $\begin{array}{c}\text { Relativamente } \\
\text { satisfecho }\end{array}$ & Indiferente, & $\begin{array}{c}\text { Relativamente } \\
\text { insatisfecho }\end{array}$ & $\begin{array}{l}\text { Muy } \\
\text { insatisfecho. }\end{array}$ \\
\hline $\begin{array}{l}\text { Respecto la metodología } \\
\text { utilizada. }\end{array}$ & $80 \%$ & $15 \%$ & $5 \%$ & $0 \%$ & $0 \%$ \\
\hline $\begin{array}{l}\text { Respecto a las guías de } \\
\text { aprendizaje }\end{array}$ & $81 \%$ & $15 \%$ & $3 \%$ & $1 \%$ & $0 \%$ \\
\hline $\begin{array}{l}\text { Respecto al aprendizaje } \\
\text { logrado }\end{array}$ & $90 \%$ & $10 \%$ & $0 \%$ & $0 \%$ & $0 \%$ \\
\hline $\begin{array}{l}\text { Respecto al proyecto en } \\
\text { general }\end{array}$ & $5 \%$ & $0 \%$ & $0 \%$ & $0 \%$ \\
\hline
\end{tabular}

De la tabla 2 se observa la buena acogida que tuvo el proyecto de clase invertida, en donde el $95 \%$ de los estudiantes que participaron en el proyecto indicaron una buena aceptación de este y evidenciaron que el material y la metodología utilizados fueron útiles en el su proceso de enseñanza-aprendizaje.

\section{DISCUSIÓN}

A través de la intervención metodológica con el proyecto de clase invertida, se logró aumentar los promedios de notas y tasas de aprobación, tal como se verifica en la tabla 1 y figura 3 con, incluso con una disminución de la asistencia de los estudiantes a clases. Esto último es consecuente ya que la metodología de clase invertida "permite a los estudiantes moverse en un escenario que lleva de la atención en un aula a determinados conocimientos, a la interpretación de los conocimientos de manera autónoma" (Perdomo, 2016), y por lo tanto se generan aprendizajes de mayor significancia y más duraderos. Además, la clase invertida 
incentiva un rol más activo y protagónico de los estudiantes en su propio aprendizaje, ya que el profesor asume un rol de orientador y/o asesor de los estudiantes (Tourón et al., 2014; Perdomo, 2016).

En la sociedad de la información, la tecnología es un recurso útil en diversas actividades del quehacer humano, y más aún en educación (Rodríguez et al., 2017). Tal como resalta Gordon et al. (2018), la alta demanda de educación en línea está definiendo las estrategias de futuro de las instituciones de educación alrededor del mundo. Aun cuando Crompton y Burke (2018) señalan una falta de investigación en el real impacto de la tecnología móvil para con la educación, Heflin et al. (2017) indica un impacto positivo en el aprendizaje colaborativo de los estudiantes con la utilización de la tecnología móvil. Así, también existen experiencias positivas en el proceso de enseñanza-aprendizaje con la utilización de tecnología móvil, para geometría con el uso de computador y herramientas tecnológicas tales como (Fonseca-Pérez y GamboaGrau, 2010) quienes presentan experiencias positivas en el uso de tecnología en la enseñanza de geometría en colegios de Cuba, (Diaz-Nunja et al., 2018) que describen experiencias positivas del uso de la herramienta GeoGebra para la enseñanza de la geometría, (Ferreira de Souza, 2017) que muestra experiencias en colegios para el aprendizaje de la geometría en tres dimensiones con GeoGebra3D, (Calderón, 2015) quien destaca el aprendizaje de geometría con el uso de realidad aumentada, y el trabajo de Bano et al. (2018) quien describe positivas experiencias del uso de la tecnología móvil para el aprendizaje de las matemáticas en educación. Por todo lo anterior, las herramientas tecnológicas son fundamentales en el proceso de enseñanza-aprendizaje de la geometría lo que es una invitación mayor para la realización de sesiones de clase invertida con el apoyo de herramientas tecnológicas para el desarrollo de competencias de geometría.

\section{CONCLUSIONES}

El objetivo de este proyecto era lograr que los estudiantes-trabajadores pudieran desarrollar competencias y aprendizajes esperados a pesar de la inasistencia, lo que fue posible por la aplicación del modelo de clase invertida con guías de aprendizaje y videos para que estos estudiantes pudieran trabajar y ver en su tiempo libre para lograr aprendizajes significativos y, con el desarrollo de un aprendizaje autónomo y regulado, obtuvieran un impacto positivo en su retención, aprobación y promedio de notas. Los estudiantes a través de entrevistas catalogaron positivamente la experiencia ya que lograron estudiar a su propio ritmo, repitiendo la clase si fuera necesario y teniendo acceso a la misma desde cualquier lugar, lo que aumentó su motivación y satisfacción con la asignatura de Geometría, favoreciendo el aprendizaje activo y significativo. Los videos de reforzamiento sirvieron con el mismo propósito que las guías de aprendizaje, pero esta vez, el estudiante pudo ver al profesor resolver los ejercicios que se estudiaron en clases; su profesor realizó el paso a paso de cada ejercicio. Así, cada estudiante fue capaz de dilucidar dudas que no logró abordar en clases.

\section{REFERENCIAS}

Aliksia, K., Dwi Mardiyana, U. y Pramudya, I., Analysis of Junior High School Students' Difficulty in Resolving Rectangular Conceptual Problems, doi: 10.1063/1.4995135, American Institute of Physic AIP Conference Proceedings, 1868 (1), Rusia (2017)

Arráez, G., Lledó, A., Gómez, M. y Lledó, G., La clase Invertida en la Educación Superior: Percepciones del Alumnado, doi: 10.17060/ijodaep.2018.n1.v2.1197, International Journal of Developmental and Educational Psichology, 1(1), ISSN 0214-9877 (2018)

Bano, M., Zowghi, D. y otros 3 autores, Mobile Learning for Science and Mathematics School Education: A Systematic Review of Empirical Evidence, doi: 10.1016/j.compedu.2018.02.006, Computers \& Education, 121, 30 - 58, Febrero (2018)

Bergmann, J. y Sams, A., Flipped Learning, doi: 10.1111/bjet.12368, British Journal of Educational Technology, 46(6), Wiley-Blackwell Academic Journals, (2014)

Bergmann, J. y Sams, A., Flipped Learning: Gateway to Student Engagement, ISBN 978-1-56484-344-9, Center for Educational Policy Studies Journal, 7(3), 173 - 176, USA (2017)

Berenguer, C., Acerca de la Utilidad del Aula Invertida o Flipped Classroom, XIV Jornadas de Redes de Investigación en Docencia Universitaria, Instituto de Ciencias de la Educación, Universidad de Alicante, 1466 - 1480, Alicante, España (2016)

Boccaccio, A., Uva, A. E. y otros 3 autores, Geometry Design Optimization of Functionally Graded Scaffolds for Bone Tissue Engineering: A Mechanobiological Approach, doi: 10.1371/journal.pone.0146935, PLoS ONE 11(1): e0146935 (2016)

Calderón, F., Realidad Aumentada Aplicada a la Enseñanza de la Geometría Descriptiva, doi: 10.4206/aus.2015.n18-04, AUS, 18(04), 18 - 22, Universidad Austral de Chile, Valdivia, Chile, Diciembre (2015)

Camacho-Yáñez, I., Gómez-Zermeño, M. y Pintor-Chávez, M., Competencias Digitales en el Estudiante Adulto Trabajador, Revista Interamericana de Educación de Adultos, 37(2), 10-24 (2015)

Clement, D., Geometric and spatial thinking in early childhood education, doi: 10.4324/9781410609236, Engaging Young Children in Mathematics: Standards for Early Childhood Mathematics Education, 261 - 297, Enero (2004) 
Crompton, H. y Burke, D., The use of Mobile Learning in Higher Education: A Systematic Review, doi: 10.1016/j.compedu.2018.04.007, Computers \& Education 123, Marzo (2018)

Crompton, H., Grant, M. R. y Shraim, K. Y. H., Technologies to Enhance and Extend Children's Understanding of Geometry: A Configurative Thematic Synthesis of the Literature, Educational Technology \& Society, ISSN 1436-4522, $21(1), 59-69$ (2018)

Diaz-Nunja, L., Rodríguez-Sosa, R. y Lingán, S. K., Enseñanza de la geometría con el software GeoGebra en estudiantes secundarios de una institución educativa en Lima, doi: 10.20511/pyr2018.v6n2.251, Propósitos y Representaciones, 6(2), 217 -251, Julio - Diciembre (2018)

Dimmed, J. K. y Herbert, P. G., What Details Do Teachers Expect from Student Proofs? A Study of Proof Checking in Geometry, Journal for Research in Mathematics Education, 49(3), 261 - 291, Mayo (2018)

Ferreira de Souza, G. M., Uso de GeoGebra3D en la Enseñanza de Geometría Espacial, Tesis de Maestría Profesional en Matemáticas en Red Nacional, Universidad Federal de Juiz de Fora, Instituto de Ciencias Exactas, Brazil (2017)

Fonseca-Pérez, J. J. y Gamboa-Grau, M. E., La enseñanza de la Geometría Asistida por Computadoras: una Nueva Realidad en la Secundaria Básica, Didasc@ Lia: Didáctica y Educación, 3, 47 - 62, Cuba (2010)

Friz, M., Panes, R., Salcedo, P. y Sanhueza, S., El proceso de enseñanza y aprendizaje de las Matemáticas. Concepciones de los futuros profesores del sur de Chile, Revista electrónica de investigación educativa (REDIE), 20(1), ISSN 1607-4041, México, Marzo (2018)

Gamwell, L., Mathematics and Art: A Cultural History, ISBN 978-0-691-16528-8, Princeton University Press, Princeton, USA (2015)

Gordon, L. C., Gratz, E. y otros 3 autores, Utilization of Information Technology as Instructional Support in Higher Education - A Case Study, Communications of the IIMA, 16(1), California State University, San Bernardino, California, USA (2018)

Hamdam, N., Mcknight, P., Mcknight, K. y Arfstrom, K., A Review of Flipped Learning, Flipped Learning Network, Pearson, USA (2013)

Heflin, H., Shewmaker, J. W. y Nguyen, J., Impact of Mobile Technology on Student Attitudes, Engagement, and Learning, doi: 10.1016/j.compedu.2017.01.006, Computers \& Education, 107(C), 91 - 99, Abril (2017)

Monitor, Monitor Educativo, (en línea), https://monitor.iiiepe.edu.mx/. Acceso: 30 de Agosto (2019)

Novak, J., Kensington-Miller, B. y Evans, T., Flip or flop? Students' perspectives of a flipped lecture in mathematics, doi: 10.1080/0020739X.2016.1267810, International Journal of Mathematical Education in Science and Technology, 48(5), 647-658 (2017)

Orozco, D., Aplicaciones de la Geometría Plana en el campo de la Ingeniería Civil, Ingeniería Mecánica, Facultad de Mecánica, Escuela Superior de Politécnica de Chimborazo ESPOCH, Ecuador (2014)

Perdomo, W., Estudio de Evidencias de Aprendizaje Significativo en un Aula bajo el Modelo Flipped Classroom, doi: 10.21556/edutec.2016.55.618, Edutec Revista Electronica de Tecnología Educativa, 55, Marzo (2016)

Pottmann, H., Hofer, M. y Kilian, A., Advances in Architectural Geometry, ISBN 978-3-902233-03-5, Conference Proceedings, Vienna University of Technolog (2008)

Prassad, S. K. y Vijayendra, K. V., Relevance of Site Characterization in Seismic Studies, doi: 10.22607/IJACS.2017.501009, Indian Journal of Advances in Chemical Science, 5(1), 59-64 (2017)

Rodríguez, A., Ramírez, L. J. y Basile, F. R. M., Tecnologías y Educación: Su Percepción Social En Santiago de Chile, doi: 10.4067/S0718-50062017000600008, Formación Universitaria, 10(6), 67 - 76, La Serena, Chile (2017)

Rüde, U., Wilcox, K., Coffman, L. y De Stack, H., Research and Education in Computer Science, doe: 10.1137/16M1096840, SIAM Review, 60(3), 707-754 (2018)

Sjoberg-Tapia, O., Oyarzún-Lillo, F. y Ormeño-Milla, F., Determinantes en la decisión de estudiar en un plan especial universitario para trabajadores, Revista Chilena de Economía y Sociedad, 12(1), Universidad Tecnológica Metropolitana, Santiago, Chile (2018)

Szekeres, P., A course in modern mathematical physics: groups, Hilbert space and differential geometry, ISBN 0-52153645-6, Cambridge University Press, Cambridge, New York, USA (2004)

Tourón, J., Santiago, R. y Diez, A., The Flipped Classroom: Cómo Convertir la Escuela en un Espacio de Aprendizaje, Digital-Text, Barcerlona, España (2014)

Tourón, J. y Santiago, R., El Modelo Flipped Learning y el Desarrollo del Talento en la Escuela, doi: 10.4438/1988-592XRE-2015-368-288, Revista de Educación, 196-231, Madrid, España (2015)

Velishiva, D., Geometry in Engineering Education, doi: 10.1080/03043790210141979, European Journal of Engineering Education, 27(3), Taylor \& Francis (2002) 